\title{
A Multiagent Model for Supporting Tourism Policy-Making by Market Simulations
}

\author{
Arnaldo Cecchini and Giuseppe A. Trunfio \\ Laboratory of Analysis and Modelling for Planning \\ Department of Architecture and Planning - University of Sassari \\ Palazzo del Pou Salit, Piazza Duomo, 6 I07041 Alghero (SS), Italy \\ cecchini@uniss.it, trunfio@uniss.it
}

\begin{abstract}
Sustainable tourism development at a destination requires an understanding of the many interrelated factors that influence risks and returns. For this reason, operators in tourism management and public policy makers are increasingly interested in effective tools for exploring the consequences of their decisions. With this purpose, this paper describes a multiagent system which enables evaluation of the impact of some possible interventions and policies at a destination by computer simulations and provides an insight into the functioning of the market.
\end{abstract}

\section{Introduction}

As tourism is a primary industry for many geographical areas, strategic management of tourist destinations is becoming more and more important. On the other hand, the success of this economic activity strongly depends on the decisions taken by the actors in the field [1]. For example, tourism managers have to take decisions on segmentation and market positioning, product design, pricing, retail strategy and advertising, while public administrators have to implement policies regarding the accessibility of the destination and of the single attractions, the organization of events or the economic incentive of specific market segments. Given the difficulty of predicting the consequences of decisions regarding a system which involves many variables interrelated in a non-linear manner, it is recognized that models for the simulation of market dynamics can provide valuable support. Some models have already been developed for such purpose (e.g. see [23]), exploiting various techniques. In this paper we propose a multiagent model [4] which, differently from most of the existing ones, has together the following characteristics: $(i)$ it is based on spatially-situated agents; $(i i)$ the agents are heterogeneous and with memory of their past experiences; $($ iii $)$ the agents constitute a social network. As in [5], the basic assumption of the model is that tourists attempt to maximize utility from their holiday. The system is built in such a way that a tourist who is satisfied with his tourist destination is more likely to return to that destination than one who obtained lower utility. Moreover, in the model as in the real world, a satisfied tourist can influence other individuals with similar characteristics to choose the same destination, whereas an unsatisfied tourist can influence other potential tourists to avoid that destination. 


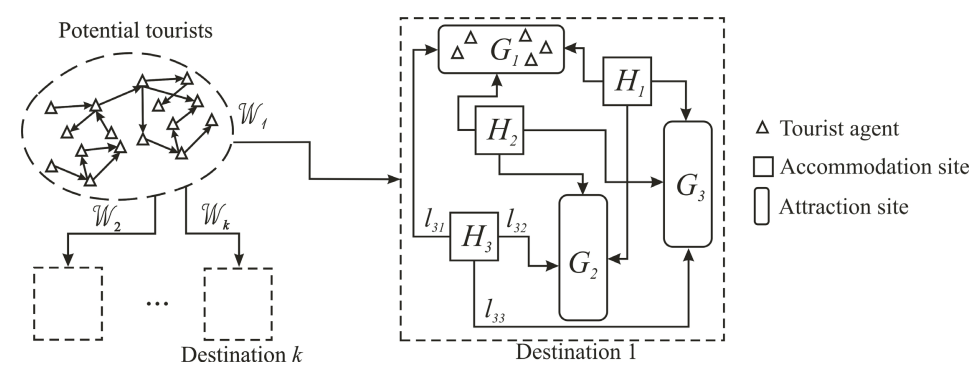

Fig. 1. The multiagent model overview

\section{Tourist Destination}

As shown in Figure 1, the main elements of the environment where agents operate are a set of tourist destinations, that is cities, towns or small geographical areas, the economy of which is to some extent dependent on revenues coming from the tourist industry. In the model a tourist destination is represented as:

$$
\text { Destination }=\langle\mathcal{W}, \mathcal{H}, \mathcal{G}, \mathcal{L}\rangle
$$

where $\mathcal{W}=\left\{W_{1}, \ldots, W_{p}\right\}$ represents accessibility and each element:

$$
W_{i}=\langle\text { price, time, capacity }\rangle
$$

corresponds to the $i$-th means of transport available for reaching the resort (e.g. train, bus, ship, car or a combination of these) and groups together the price per person, the duration of the journey and the daily capacity, respectively.

Set $\mathcal{H}=\left\{H_{1}, \ldots, H_{n}\right\}$ of Equation 1 collects the accommodation sites:

$$
H_{i}=\langle\mathbf{x}, \text { type, quality, price, capacity }\rangle
$$

where $\mathbf{x}$ is the position in a bi-dimensional space, type is the kind of accommodation, quality is a real number belonging to the interval $[0,1]$ which summarizes the degree of excellence of the significant features of $H_{i}$, price $(t)$ provides the price per day and per person as a function of the tourist season $t_{s}$ and capacity is the number of tourists that can be simultaneously accommodated;

Set $G=\left\{G_{1}, \ldots, G_{m}\right\}$ of Equation 1 collects the attractions (i.e. places of interest that tourists visit) present at the destination, which are defined as:

$$
G_{i}=\langle\mathbf{x}, \text { type, quality, price, capacity }\rangle
$$

where $\mathbf{x}$ is the position in a bi-dimensional space, type is the kind of attraction, quality $\in[0,1]$ is a synthetic indicator of the quality of the significant features of $G_{i}$ (e.g. view, water and sand quality for an uncontaminated beach or the quality of the services and facilities for an equipped beach), price ( $t$ ) provides the entrance price per person given season $t_{s}$ and capacity is the number of people who can simultaneously enjoy $G_{i}$. 
Set $\mathcal{L}$ of Equation 1 contains links:

$$
l_{i j}=\left\langle H_{i}, G_{j}, \text { type, price, time }\right\rangle
$$

specifying the money and time costs which are required for reaching the attraction site $G_{j}$ from the accommodation site $H_{i}$, using the means of transport specified by the attribute type. Thus, the elements of $\mathcal{H}$ and $\mathcal{G}$ are the edges of a graph representing the environment in which the model agents operate.

For each destination, an accessibility agent answers the tourist agent's requests, that is, he provides the first available departure date, given the period, the access mode and the duration of the stay. Besides, each accommodation site $H_{i}$ and attraction site $G_{i}$ holds a manager agent that has three main tasks: $(i)$ to keep a schedule of admittance in order to accept or deny booking requests on the basis of the site's capacity, by interacting with tourist agents $(i i)$, to answer questions about the current state of the site (e.g. the number of people currently hosted) (iii), to adapt his prices to the demand coming from tourists.

\section{Tourist Agent}

Each tourist, who it is assumed takes one vacation per year, has the main aim of maximizing his utility and is capable of remembering the experiences had at a destination. In addition, he can receive information from other tourists or from advertising messages. More formally, the tourist agent is defined as:

$$
A=\langle\mathcal{P}, \mathcal{S}, \mathcal{M}, \mathcal{E}, \mathcal{V}, \mathcal{B}\rangle
$$

In the latter Equation $\mathcal{P}$ is a set of properties that influences the tourist's behaviour (e.g. Age, Gender, Family size, Income, Education).

Set $\mathcal{S}=\left\{t_{0}, T^{(h)}, A c c, A t t\right\}$ collects some properties that concur in defining the agent's state. In particular, $t_{0}$ is the instant of the start of the journey, $T^{(h)}$ is the holiday duration, while $A c c$ and $A t t$ are two links which, during the temporal interval $\left[t_{0}, t_{0}+T^{(h)}\right]$, indicate the accommodation site and the attraction site currently holding the agent, respectively.

Set $\mathcal{V}$ in Equation [ constitutes the information concerning potential tourist destinations possessed by the agent. Each piece of information is represented as:

$$
V=\left\langle\text { Dest, St, } p_{G}, \mathcal{G}_{V}\right\rangle
$$

where Dest refers to the destination, $S t$ is the information strength, $p_{G}$ is an estimate of the cost per day of a stay excluding accommodation and $\mathcal{G}_{V}$ id the set of attractions known by the agent in Dest. In each element $g_{i}=\left\langle G_{k}, n_{i}, \alpha_{i}\right\rangle \in$ $\mathcal{G}_{V}$, the value of $n_{i}$ is the number of visits of the attraction $G_{k}$ in a previous stay in Dest while $\alpha_{i}$ is an evaluation parameter assigned to the same attraction. The quantity $S t \in[0,1]$ represents a measure of information reliability perceived by the agent (e.g. $S t=1$ in the case of information originating from direct knowledge). During the simulation, information strength $S t$ varies according 
to two mechanisms: at each time-step $k$ it decreases according to the relation $S t^{(k+1)}=\sigma S t^{(k)}$, with $\sigma \in[0,1]$ and it changes in the case the agent receives new elements of information about Dest.

Set $\mathcal{E}=\left\{E_{1}, \ldots, E_{q}\right\}$ in Equation 6 groups together elements $E_{i}$ defined as:

$$
E_{i}=\left\langle A t t_{i}, n_{i}^{(s)}, n_{i}^{(f)}, p_{i}, r_{i}, \alpha_{i}\right\rangle
$$

representing the agent's memory of attractions experienced during a vacation. In particular, $A t t_{i}$ is the link to the attraction, $n_{i}^{(s)}$ is the number of accesses to $A t t_{i}, n_{i}^{(f)}$ is the number of failures in accessing (e.g. an access request may be refused when $A t t_{i}$ is full), $p_{i}$ is the total expenditure made for the attraction, $r_{i}$ is the sum of all ratings attributed to each of the $n_{i}=n_{i}^{(s)}+n_{i}^{(f)}$ requests, $\alpha_{i}$ represents the overall evaluation which is attributed by the agent to the $n_{i}$ experiences on the whole. For $t=t_{0}$ (i.e. the holiday start) the set $\mathcal{E}$ is empty. During the holiday, the agent fills the set $\mathcal{E}$ of experiences with evaluations $\alpha_{i}$. At the holiday end, values $n_{i}^{(s)}$ and $\alpha_{i}$ are transferred in the corresponding variables of the set $V$ related to the destination (see Equation 7 ).

Set $\mathcal{M}$ in Equation 6 collects links to other potential tourists. As explained below, the network of tourist agents determines the exchange of information related to potential tourist destinations.

The agent's behaviour is defined by the actions collected in set $\mathcal{B}$ of Equation 6 During the simulation, agents have to make decisions about actions to undertake (e.g. which destination to visit). We assume that such decisions are made probabilistically, according to a logistic model [6. In particular, given a choice set $\mathcal{C}$, the agent associates a utility value $U\left(c_{i}\right)$ to each alternative $c_{i} \in \mathcal{C}[7$. The utility values are then transformed into probabilities $\operatorname{Pr}\left(c_{i} \mid \mathcal{C}\right)=e^{U\left(c_{i}\right)} / \sum_{j=0}^{\sharp \mathcal{C}} e^{U\left(c_{j}\right)}$, where $\sharp \mathcal{C}$ is the number of alternatives. The actual choice is made comparing a drawn random number in $[0,1]$ with the cumulative probabilities $\sum_{j=0}^{i} P\left(c_{j} \mid \mathcal{C}\right)$ associated to the alternatives $c_{i}$. In some cases, the choice account for a satisfier threshold $U_{t h}$, that is, in the choice set $\mathcal{C}$, the alternatives $c_{k}$ with $U\left(c_{k}\right)<U_{t h}$ are not considered by the agent.

If the current time $t$ is greater than $t_{0}+T^{(h)}$, at each time-step of the simulation and with probability $p_{b}$, the tourist agent tries to book a new holiday. The algorithm proceeds by choosing first the preferred season and the holiday duration $T^{(h)}$, then the trip destination together with the means of transport and the accommodation site. At the end the agent tries to book for a specific departure date $t_{0}$. In the follows only the most relevant agent's actions are illustrated.

Choice of destination, accommodation and means of transport. When the agent decided to take a vacation, that is, the period of the year and the duration $T^{(h)}$ have been chosen, for each piece of information regarding potential destinations possessed by the agent (i.e. contained in $\mathcal{V}$ ), and for each combination $\left\langle W_{k}, H_{j}\right\rangle$, the agent computes two utility functions:

$$
U_{D}=\frac{T^{(n)}\left[\sum_{i=1}^{m}\left(1+S t \alpha_{i}\right) \beta_{i}\left(n_{i}\right) q_{i}+\beta_{a c c} q_{a c c}\right]}{T(2 m+1)}
$$




$$
U_{P}=1-\frac{p_{\text {jour }}+T^{(n)}\left(p_{a c c}+p_{G}\right)}{p_{\max }(T)}
$$

which take values in the interval [0, 1]. In Equations (9) and (10):

- for each of the $m=\sharp \mathcal{G}_{V}$ different attractions known by the agent in the potential destination (i.e. in the set $\mathcal{G}$ ) there are: a function $\beta_{i}\left(n_{i}\right) \in[0,1]$ accounting for the degree of attractive influence on the agent and depending on the number $n_{i}$ of previous visits to the attraction, the coefficient $q_{i}=$ quality $(G)$ (see Equation 4) and the coefficient $\alpha_{i}$ (see Equation 7);

- St is the strength of the information on the basis of which the agent is considering the destination (see Equation 7);

$-\beta_{a c c} \in[0,1]$ is a parameter expressing the degree of liking of the agent for the accommodation considered, having the quality $q_{a c c}=$ quality $\left(H_{j}\right)$;

$-T^{(n)}=T^{(h)}-\operatorname{time}\left(W_{k}\right)$ is the net time available for enjoying the stay (i.e. the total holiday duration minus the journey time);

$-p_{\text {jour }}=\operatorname{price}\left(W_{k}\right)$ is the total cost of the journey, according to the means of transport $W_{k}$ chosen, $p_{a c c}=\operatorname{price}\left(H_{j}\right)$ is the cost per day of accommodation $H_{j}$ and $p_{G}$ is the estimated cost per day of a stay at the destination considered; $p_{\max }$ is the maximum cost that the agent is willing to spend.

Agents compute their utility as the product of $U_{D}$ and $U_{P}$. The values of utility are then transformed into probabilities according to the logit model and a satisfier threshold $U_{t h}^{(D)}$ is used. for the choice.

Given the destination Dest, the access mode $W_{k}$ and the accommodation $H_{j}$, the trip start date $t_{0}$ is determined by interacting with the accessibility agent and the manager agent (see Section 2).

The holiday experience. During the stay the tourist's agent attempts to maximize utility from his holiday. In particular, at $t=t_{0}$ the set $\mathcal{E}$, which represents the history of the holiday, is empty. The agent's accommodation site being $H_{i}$, every time-step of the simulation the agent chooses an attraction $G_{j} \in \mathcal{G}$ by using the utility function:

$$
\begin{aligned}
U_{G} & =\frac{1}{2}\left(1-\frac{\pi\left(G_{j}, l_{i j}\right)}{\pi_{\max }\left(G_{j}\right)}\right)(1+\alpha) \beta q \\
\pi\left(G_{j}, l_{i j}\right) & =\operatorname{price}\left(G_{j}\right)+\operatorname{price}\left(l_{i j}\right)+\mu \operatorname{time}\left(l_{i j}\right)
\end{aligned}
$$

where $\beta$ and $q$ have the same meaning so that in Equation 9 , while the parameter $\alpha$, which is kept in memory by the agent as stated in Equation 8 expresses an overall evaluation relative to the attraction site on the basis of previous experiences of the same stay, $\mu$ is the cost of the time which depends on the agent's characteristics $\left[8\right.$ and $\pi_{\max }\left(G_{j}\right)$ is the maximum cost that the agent is willing to spend for enjoying the attraction. The choice is made transforming values (11) into probabilities and using a satisfier threshold $U_{t h}^{(G)}$. Let Att be the attraction chosen, the corresponding entry $E \in \mathcal{E}$ (see Equation 8) is updated in order to reflect the new experience. In particular: 

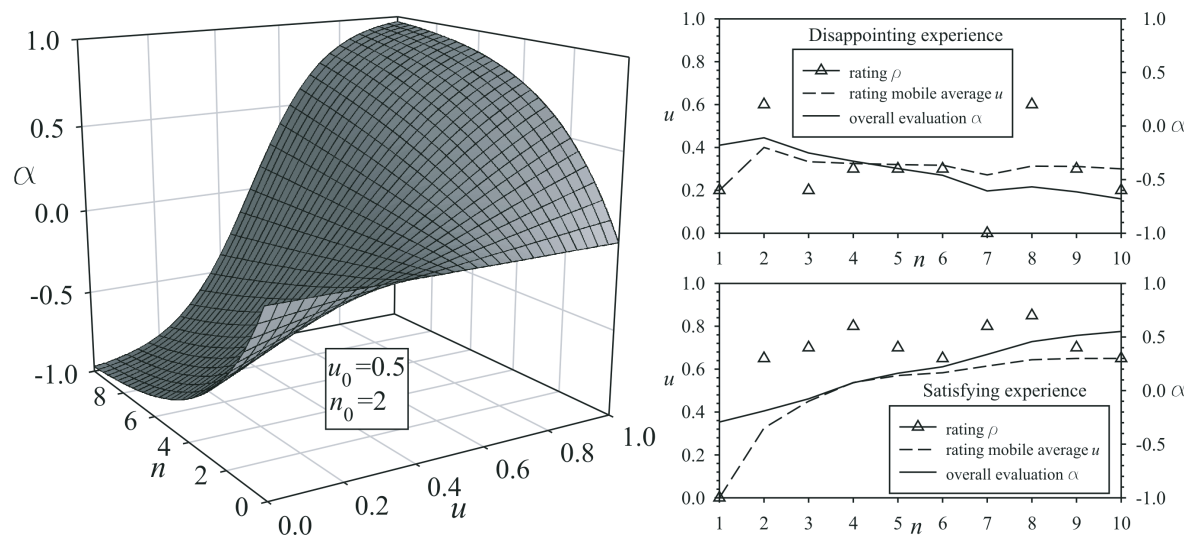

Fig. 2. The evaluation function used by the tourist agent

1. $n^{(s)}$ (or $n^{(f)}$ in the case the request of access is refused) is incremented by one, the total expenditure $p$ is updated adding the new value $\pi\left(G_{j}, l_{i j}\right)$ and the total rating $r$ is updated with the new rating $\rho \in[0,1]$;

2. the evaluation $\alpha(u, n)$ of the attraction site is computed as a function of the average rating $u=r / n$ and of the number of requests $n=n^{(s)}+n^{(f)}$.

The evaluation function $\alpha(u, n)$ is defined as:

$$
\alpha(u, n)=\frac{e^{\frac{u}{u_{0}} \ln b(n)}-b(n)}{e^{\frac{u}{u_{0}} \ln b(n)}+b(n)}, \quad \text { with } \quad b(n)=2 e^{\frac{n}{n_{0}} \ln 2}-1
$$

where the parameters $n_{0}$ and $u_{0}$ have the following meaning: $(i)$ if the average rating $u$ is still null after $n_{0}$ access requests, this corresponds to an overall evaluation of $\alpha=-0.5$, that is, to the halving of the next estimation of the utility component $U_{E}$ for that attraction site; $(i i) u_{0}$ is the average rating corresponding to the neutral evaluation $\alpha=0.0$. Figure 2 reports two examples of agent's experience for an attraction site using Equations 13. It can be seen that ratings above $u_{0}$ contribute, throughout the history of the experience, to increase the overall evaluation $\alpha$, making the experience satisfying. On the contrary, a sequence of ratings below $u_{0}$ produces, in agent convincement, a bad overall evaluation of the attraction site. The rating $\rho$ should reflect the actual agent's experience at the attraction site. Thus, its determination depends on both the agent's interactions and the interaction between agents and the environment. At the end of the holiday, total expenditures $p$, number of accesses $n_{i}^{(s)}$ and evaluations $\alpha_{i}$ contained in the agent's memory $\mathcal{E}$, are transformed into updated pieces of information for the set $\mathcal{V}$.

Influencing other individuals. As shown in Figure 1, potential tourists constitute a social network in the sense that each agent can influence the decisions of other agents. The latter are the ones pointed by the links contained in the set $\mathcal{M}$ of each agent. The network is randomly initialized at the simulation start by 
Table 1. The main characteristics of the focused destination

\begin{tabular}{lcccc}
\hline Id & type & \multicolumn{3}{c}{ quality price capacity } \\
\hline \multicolumn{5}{c}{ Accommodation sites } \\
\hline$H_{1-5}$ & four-star hotel & 0.8 & 130 & 200 \\
$H_{6-15}$ & three-star hotel & 0.8 & 80 & 240 \\
$H_{16-35}$ & bed \& breakfast & 0.6 & 35 & 10 \\
\hline \multicolumn{5}{c}{ Attraction sites } \\
\hline$G_{1-4}$ & beach & 0.6 & 2 & 500 \\
$G_{5-7}$ & equipped beach & 0.6 & 10 & 600 \\
$G_{8}$ & historical center & 0.5 & 5 & 5000 \\
$G_{10}$ & museum & 0.8 & 4 & 600 \\
\hline \multicolumn{5}{c}{}
\end{tabular}

\begin{tabular}{lccc}
\hline \multicolumn{4}{c}{ Accessibility } \\
\hline Id & price & time capacity \\
\hline$W_{1}$ & 300 & 3 & 360 \\
$W_{3}$ & 100 & 12 & 1000 \\
$W_{3}$ & 60 & 4 & 180 \\
\hline
\end{tabular}

assigning for each agent the number of elements in $\mathcal{M}$ according to the characteristics in $\mathcal{P}$ and by assuring that the links are predominantly directed towards agents having similar characteristics. In particular, an agent $A_{i}$ which is not in his holiday interval, selects with probability $p_{e}$ a group of neighbouring agents. Then, to each of the selected agents $A_{j}$, agent $A_{i}$ communicates one of his pieces of information $V^{(i)} \in \mathcal{V}$ which is probabilistically selected. When the agent $A_{j}$ receives the element of information $V^{(i)}$, he updates his corresponding element $V^{(j)}$ which refers to the same destination as:

$$
\hat{S} t^{(j)}=\max \left(S t^{(j)}, \lambda_{i j} S t^{(i)}\right) \quad \text { and } \quad \hat{\alpha}_{k}^{(j)}=\left(\alpha_{k}^{(j)}+\lambda_{i j} S t^{(j)} \alpha_{k}^{(i)}\right) / 2
$$

where $\hat{V}^{(j)}$ is the updated information and the coefficient $\lambda_{i j} \in[0,1]$ is the degree of trust of the agent $A_{i}$ in the eyes of $A_{j}$.

\section{A Preliminary Test}

The model, implemented in $\mathrm{C}++$ language, was tested with a hypothetical but plausible scenario. A set of potential tourists was initialized with 100.000 agents having random characteristics and relationships. Five destinations, each having various access modes, accommodation and attraction sites, were included in the artificial marketplace. The time-step size was set at 12 hours and it was assumed each tourist would visit one attraction per step during vacations. The destination highlighted was composed in particulary of elements $H_{i}$ and $G_{i}$ having the characteristics shown in Table 1, For each couple $H_{i}$ and $G_{j}$ three links $l_{i j}$, were generated with random properties appropriate to represent walking, moving by bus and by taxi, respectively. The attractions present at the destinations were constantly populated by local visitors (i.e. visitors not accommodated at any of the sites in $\mathcal{H}$ ) for one half of their capacity.

The test presented here was aimed at assessing the influence of a modification in accessibility $\mathcal{W}$. To this end, two groups of four runs were executed averaging the probabilistic results. Each run was composed of 2920 steps, corresponding to four years. In the first group the set $\mathcal{W}$ for the destination under study was composed of the two elements $W_{1}$ and $W_{2}$ shown in Table 1 . In the second group, the element $W_{3}$ shown in Table 1, which represents a daily flight of a low-cost company, 

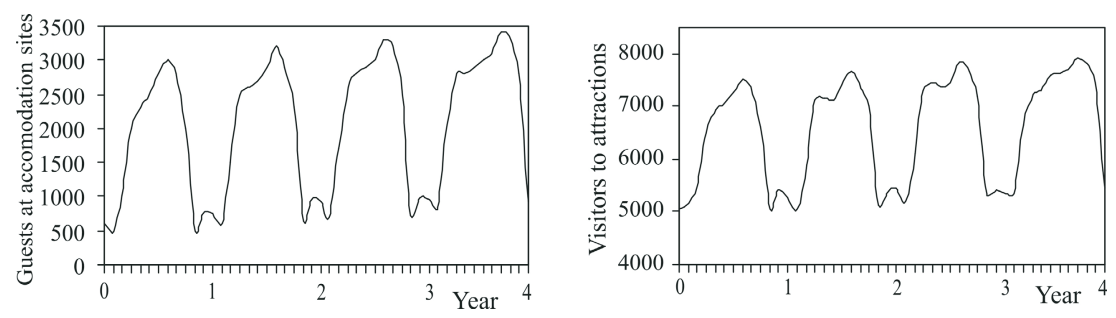

Fig. 3. Effects of the introduction of a low-cost daily flight

was added to the set $\mathcal{W}$ at the end of the first year. The results are illustrated in Figure 3. where both the effects in terms of guests at accommodation sites and visitors to attraction sites are reported. According to the model, the effects in terms of number of visiting tourists affect both the peaks in high season and, to a lower extent, the visitors in low season. The increment of tourists grows in the second year by the introduction of $E_{3}$ and tends to become stable after two years.

\section{Conclusions and Future Work}

The model allows evaluation of the effect of advertising messages, the effect of pricing strategies, market segmentation approaches, introduction or enhancement of new attractions or the interrelated consequences of many simultaneous actions. On the other hand, a drawback is represented by the difficulties which may arise in the initial setup of the model for an actual destination. Indeed, this phase requires a time-consuming activity involving the definitions of parameter values and the validation by comparison with real historical trends for similar situations. Future work will focus on contextualizing the model in a real geographical area, for which sufficient data are available.

\section{References}

1. Sainaghi, R.: From contents to processes: Versus a dynamic destination management model (DDMM). Tourism Management 27 (2006) 1053-1063

2. Velthuijsen, J.W., Verhagen, M.: A simulation model of the dutch tourism market. Annals of Tourism Research 21(4) (1994) 812-827

3. Kandelaars, P.: A dynamic simulation model of tourism and environment in the yucatan peninsula. Working papers, Int. Inst. for Applied Systems Analysis (1997)

4. Wooldridge, M.: An Introduction to MultiAgent Systems. John Wiley \& Sons (2002)

5. Hernández-López, M., Cáceres-Hernández, J.J.: Forecasting tourists characteristics by a genetic algorithm with a transition matrix. Tourism Management 28 (2007) 290-297

6. Sirakayaa, E., Woodsideb, A.G.: Building and testing theories of decision making by travellers. Tourism Management 26 (2005) 815-832

7. Russel, S., Norvig, P.: Artificial Intelligence: A Modern Approach. Prentice-Hall (1995)

8. Meignan, D., Simonin, O., Koukam, A.: Multiagent approach for simulation and evaluation of urban bus networks. In: 5rd AAMAS Conference 